\title{
SPACE- AND TIME-RESOLVED RAMAN AND BREAKDOWN SPECTROSCOPY: ADVANCED LIDAR TECHNIQUES
}

\author{
Gurlui Silviu $^{1 *}$, Cazacu Marius Mihai ${ }^{1,2}$, Timofte Adrian ${ }^{1,3}$, \\ Rusu Oana ${ }^{1}$, Bulai Georgiana ${ }^{1,4}$, Dan Dimitriu ${ }^{1}$ \\ ${ }^{I}$ Alexandru Ioan Cuza University of Iasi, Faculty of Physics, Atmosphere Optics, Spectroscopy and \\ Lasers Laboratory, 700506 Iasi, Romania, *sgurlui@uaic.ro \\ ${ }^{2}$ Gheorghe Asachi Technical University of Iasi, Department of Physics, 700050 Iasi, Romania, \\ marius.cazacu@tuiasi.ro \\ ${ }^{3}$ National Meteorological Administration, Regional Forecast Center Bacau,3 Timpului Str., Bacau, \\ Romania), timofte.adrian@gmail.com \\ ${ }^{4}$ CERNESIM, Alexandru Ioan Cuza University of Iasi, 700506 Iasi, Romania
}

\begin{abstract}
DARLIOES - the advanced LIDAR is based on space- and time-resolved RAMAN and breakdown spectroscopy, to investigate chemical and toxic compounds, their kinetics and physical properties at high temporal (2 ns) and spatial (1 $\mathrm{cm})$ resolution. The high spatial and temporal resolution are needed to resolve a large variety of chemical troposphere compounds, emissions from aircraft, the self-organization space charges induced light phenomena, temperature and humidity profiles, ice nucleation, etc.
\end{abstract}

\section{INTRODUCTION}

Introduction of new technologies to investigate the physic-chemical phenomena related to the Earth's climate changes is highly required due to the increased number of pollutant sources (widely major relevant potential in the global warming effect) and their adverse greenhouse effects caused by compounds such as carbon dioxide, nitrous oxide, sodium chlorine, methane and high global warming potential gases like perfluorocarbons, sulfur hexafluoride, ozone substances etc. [1]. A large number of additional chemical processes involving aerosols have been postulated; however, a lack of information on the abundance, nature and reactivity of the various aerosol types (e.g., dust, soot, sulfate, nitrate, organic) has hindered an accurate assessment [2].

In the last decades, especially due to more frequent extreme weather events, worldwide Environmental Research concentrated its efforts toward development of climate change prediction and scenarios to improve the regional climate change predictions. It became necessary to use all available expertise and instrumentation in order to improve model representation of physical chemical and biological processes, to assess impacts of climate changes and to quantify and reduce the uncertainties in evaluation of the hydrological cycle and impacts on natural resources, socio-economic systems and human health. In order to accomplish this objective, a large amount of experimental data must be collected, processed, evaluated and analyzed

The study of the aerosols atmosphere optics of ultra-trace amounts such as organic or in-organic compounds plays a key impact factor in the earth physics also. To identify the physico-chemical species behaviours, their concentrations and human health risk, influence on climate changes, different sensors, both ground-based and satellites optical instruments are extensively used today. Moreover, the satellite spectral instruments, providing continuous, long-term data sets, environmental forecasting and global climate studies, have in particular a great potential to detect most of the atmospheric pollutants, water amount, brightness temperatures, etc. Thus, remote sensing from satellite evidences global coverage in latitude and longitude, but it may be hard to achieve any altitude selectively: time (magnitude of seconds)- space (magnitude of few $\mathrm{km})$ resolution of measurements which are very limited in order to measure and understand fast chemical and optical phenomena induced from various physical atmospheric parameters.

\section{DARLIOES SYSTEM}

In order to better understand the fundamental of some critical physico-chemical transformation of 
the atmosphere compounds but also for applications point of view, a new optical instrument was developed in the framework of the STAR-ROSA. As in many scientific fields, in the atmosphere science, a major progress is driven through the introduction of new or improved techniques involving both analytical laboratory experiments and field campaigns. For example, the project "Fast laser imaging, detection and ranging of aerosol emissions in aircraft plumes"(DARLIOES) had the main objective to develop a new technique where coupled high resolution laser, passive and active remote optical emission spectroscopy instruments are used in order to study different aerosol emissions in aircraft plumes [DARLIOES was supported by the Romanian Space Agency (ROSA) within Space Technology and Advanced Research (STAR) Program (Project no. 98/ 29.11.2013), http://spectroscopy.phys.uaic.ro/darlioes.html]. Some advantage of the DARLIOES instrument are the following: time-space resolved temperature and humidity profiles (Raman scattering of oxygen $353 \mathrm{~nm} \& 354 \mathrm{~nm}$; real-time monitoring tool to measure concentration of sulphur dioxide injected in the troposphere from the volcanic eruption ash, water profiles and their dynamics, acid rain, chemical transformation/physical properties; DIAL $\mathrm{H}_{2} \mathrm{O}$ and $\mathrm{SO}_{2}$; UV fluorescence using the two stronger absorption emission lines of $218.9 \mathrm{~nm}$ and 220.8 $\mathrm{nm}$, respectively; water vibrational Raman scattering at $407 \mathrm{~nm}$; ice nucleation variability, physical and chemical impact, the influence upon the mixed-phase cloud; dust mineralogical composition dynamics influences upon the ice nucleation; Raman scattering of oxygen - $353 \mathrm{~nm}$ $\& 354 \mathrm{~nm}$; fast ICCD imagery (IR spectrum) of ice nucleation pattern clouds; studies of the ice nucleation and propagation in plants; UV fluorescence of PAH \{Benzo(ghi)perylene Excitation (Ex-301 nm/ Emission Em-420 nm); Naphthalene (Ex-275 nm/ Em-325 nm); Anthracene (Ex-255 nm/Em-375 nm); Pyrene (Ex-270 nm/ Em-420 nm), etc) $\}$.

Approaches of combining lidar and sun photometer data have been undertaken recently. A combination of spectrally resolved optical depth measured with sun photometer and backscatter coefficients measured with Raman lidar was used to explore the possibility of deriving important particle variables such as volume and surface-area concentration, effective radius, and singlescattering albedo [3]. Such synergetic retrieval results in additional enhancements in derived aerosol properties because the back-scattering observations by lidar improves the sensitivity in columnar property analysis of aerosol, while radiometric observations provide sufficient constraints on aerosol type and concentrations that are generally missing in lidar signals. Remote sensing methods, both active and passive, proved to be fruitful and convenient. A number of developed and launched space instruments [4], [5] provide global monitoring of aerosol properties [6], [7]. The retrieval of the physical, chemical and spectral aerosol properties from the optical measurements is a highly non-trivial and challenging task. It is often not possible to extract full information about the aerosol field from observations at fixed sites and for a limited set of wavelength channels. Besides that, the knowledge of the small-scale physical processes within the clouds and aerosols layers is also expected to improve. The used research infrastructure is shown schematically in Figure 1. The principle of operation is based on the interaction of short and high-energy laser pulses with aerosols and clouds and on the monitoring of radiation-induced effects through high speed spectral techniques (integration time in nanosecond range).

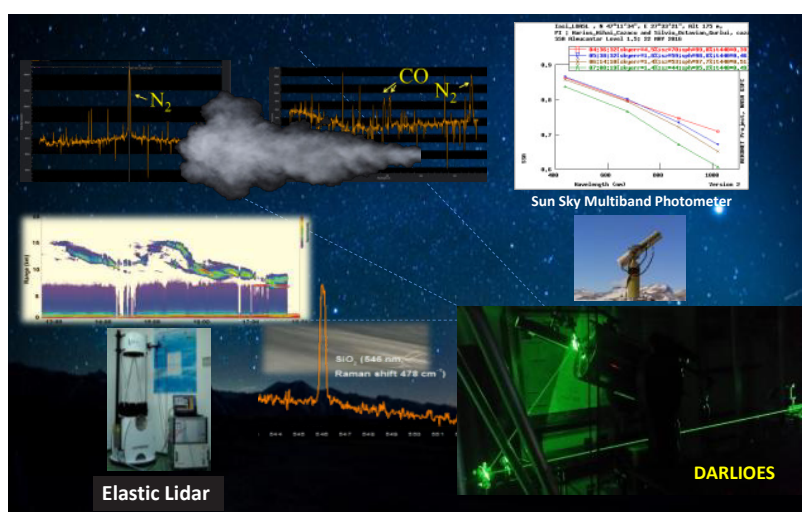

Figure 1: Approached concept of combining DARLIOES, lidar and sunphotometer

The basic lidar system described in previous papers $[8,9]$, was developed in the framework of ROmanian LIdar NETwork (ROLINET) and RADO (Romanian Atmospheric Research 3D Observatory) research projects. 


\section{RESULTS}

Preliminary measurements were performed on 27 January 2017. Meteorological data [http://www1.wetter3.de/] showed that on the 27th of January 2017, at the level of $500 \mathrm{hPa}$, field values were between 552 and 560 gpdam, highlighting the occurrence of a geopotential ridge but also thermal ridge (within 24 hours the temperature has evolved from approx. -26 to -20 degrees Celsius). Wind direction passed in 24 hours from $\mathrm{N}$ to NE. At the ground level, the high pressure system was maintained with the same value at the centre- $1035 \mathrm{hPa}$, as in the previous day. In Figure 2 shows the RCS profile recorded with the elastic lidar at $532 \mathrm{~nm}$. It can be observed that in the lower troposphere some intrusion occurs. Typically, the lidar data are correlated with complementary techniques such as the AERONET sunphotometers and with theoretical models (DREAM, HYSPLIT). Here, the preliminary results are approached by combining DARLIOES and lidar data.

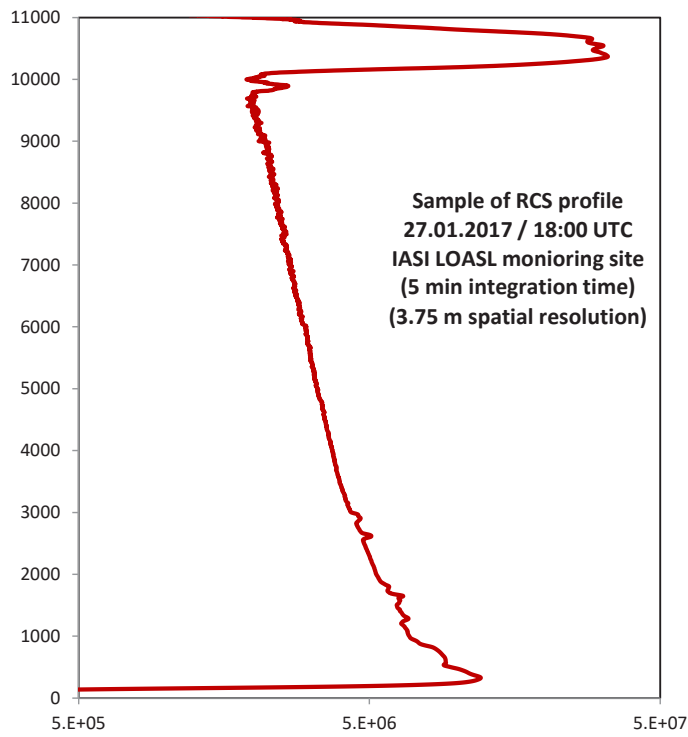

Figure 2: Sample of RCS profile

In order to analyse the atmosphere chemical compounds and their space-time dynamics optical emission spectra of the atmosphere were recorded (Figures 3 and 4).
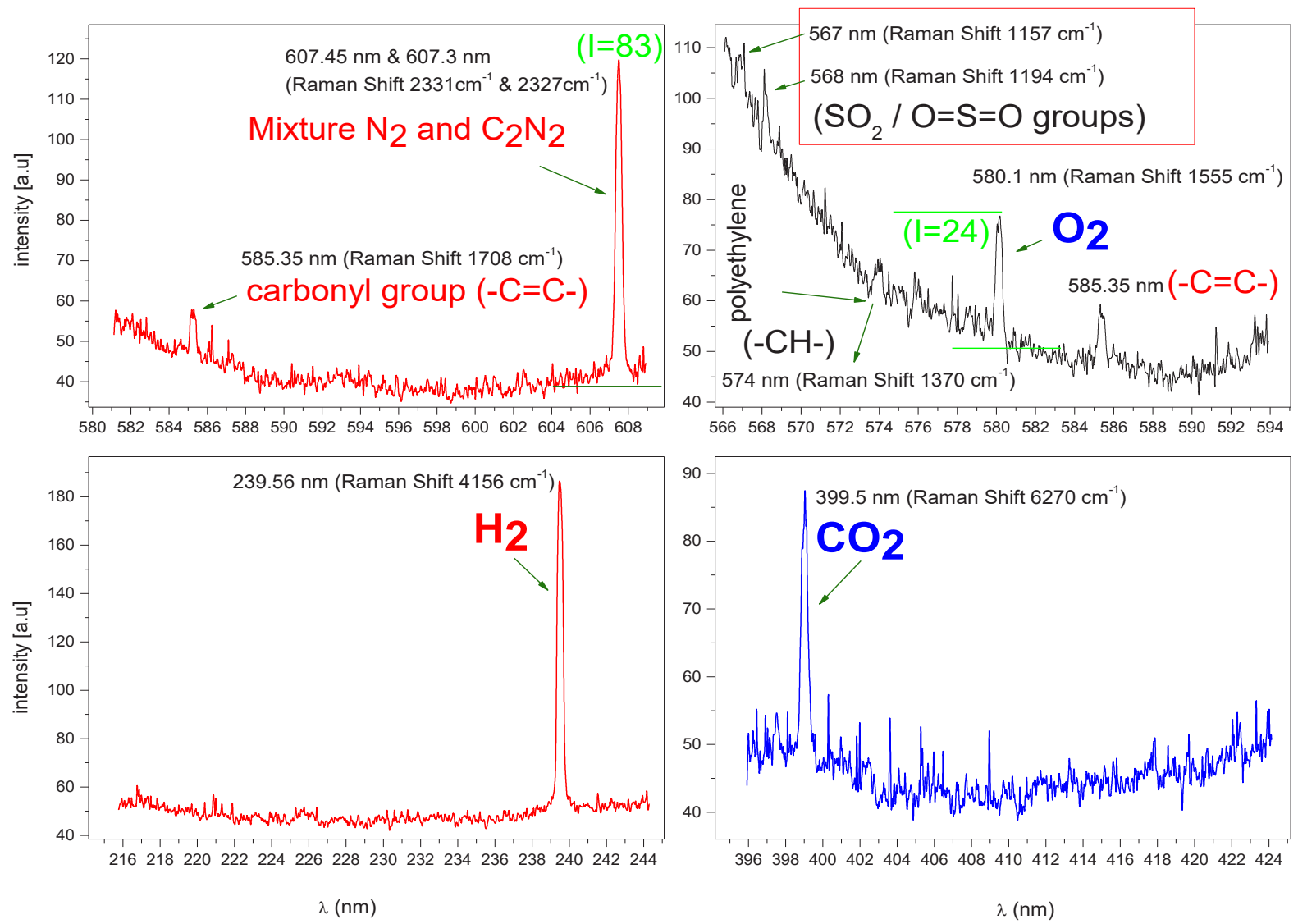

Figure 3: Time-space resolved optical emission spectroscopy of the atmosphere chemical compounds. Experimental condition: gate time 100 microseconds, delay time $=100 \mathrm{~ns}$ 


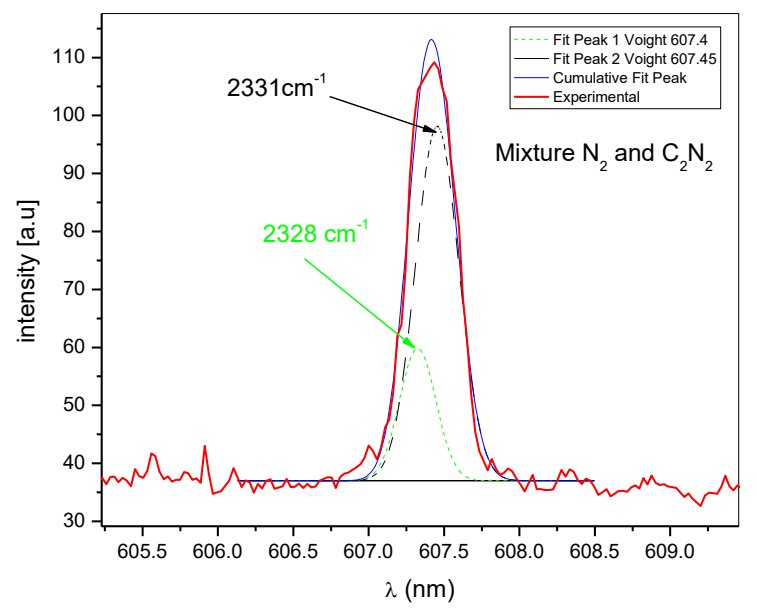

Figure 4: Time-space resolved optical emission spectroscopy of the nitrogen and cyanogens mixture.

Taking into account the wavelength of the excitation laser $(532.1 \mathrm{~nm})$ we calculated the Raman shifts for each spectral band. Therefore, we identified several known atmosphere compounds (nitrogen, oxygen, hydrogen, carbonate dioxide, sulphur dioxide, polypropylene, cyanogens, etc.). Moreover, the ratio of the two optical intensities of nitrogen and oxygen has been estimated. This is of about $\mathrm{R}=$ 3.5 , in a good agreement with the ratio of the two volume gases $(78 \%$ Nitrogen, $21 \%$ Oxygen; $\mathrm{R}=$ 3.7). In Figure 4 is showing the optical emission spectra and deconvolution of the recorded profile.

Two maxima have been evidenced at distance of $0.15 \mathrm{~nm}$. This corresponds to nitrogen molecules and nitrogen derivatives (cyanogens). This is a very interesting result of the DARLIOES because it surpasses the disadvantage of the optical filter of the classical LIDAR where the difference of the two compounds may not be differenced yet. These are preliminary data and further measurements will be done in order to estimate the dynamics of several compounds with a time resolution of up to few nanoseconds. Moreover, RCS profiles with $\sim 1$ centimeter space resolution will be recorded in order to investigate the behavior of the trace gases compound, to estimate the altitude temperature, etc.

\section{CONCLUSIONS}

In order to better understand the fundamentals of some critical physico-chemical transformations of the atmospheric compounds and for practical purpose, our study relies on a new optical power instrument. It is able to capture a fast plume airborne image ( $2 \mathrm{~ns}$ gate time) and can be used to real-time investigate the behavior of several chemical compounds at a given point of the free atmosphere, with a spatial resolution of up to 1 $\mathrm{cm}$. This new remote sensing optical instrument is based on the fast imaging and space-time resolved Raman Spectroscopy of both airborne and spaceborne plumes.

\section{ACKNOWLEDGEMENTS}

This work was financially supported by the Romanian Space Agency (ROSA) within Space Technology and Advanced Research (STAR) Program (Project no. 114/07.11.2016).

\section{References}

[1] Ciuraru, R. et al., 2011: Heterogeneous reactivity of chlorine atoms with sodium chloride and synthetic sea salt particles, Phys. Chem. Chem. Phys. 13 (43), 19460-70.

[2] IPCC, Climate Change 2013: The Physical Science Basis. 2013.

[3] Pahlow, M. et al., 2006: Retrieval of aerosol properties from combined multiwavelength lidar and sunphotometer measurements, Appl. Opt. 45 (28), 7429.

[4] Breon, F.M. et al., 2002: Scientific results from the POLarization and Directionality of the Earth's Reflectances (POLDER), Adv. Sp. Res. 30, 2383.

[5] Winker, D.M. et al., 2007: Initial performance assessment of CALIOP, Geophys. Res. Lett. 34, p. L19803,

[6] King, M.D. et al., 1999: Remote sensing of Tropospheric aerosols from Space: Past, Present, and Future, B. Am. Meteorol. Soc. 80, 2229.

[7] Kokhanovsky A. et al., 2007: Aerosol remote sensing over land: A comparison of satellite retrievals using different algorithms and instruments, Atmos. Res. 85, 372-394,.

[8] Cazacu M.M. et al., 2012: Grimsvotn Volcano: atmospheric volcanic ash cloud investigations, modelling-forecast and experimental environmental approach upon the Romanian area, J. Optoelectron. Adv. Mater. 14, (5-6), 517-522.

[9] Timofte A. et al., 2011: Romanian lidar investigation of the Eyjafjallajokull volcanic ash," Environ. Eng. Manag. J., 10 (1), 91-97. 